\title{
A Fingerprint Recognition Algorithm Combining Phase-Based Image Matching and Feature-Based Matching
}

\author{
Koichi Ito $^{1}$, Ayumi Morita ${ }^{1}$, Takafumi Aoki ${ }^{1}$, Hiroshi Nakajima ${ }^{2}$, \\ Koji Kobayashi ${ }^{2}$, and Tatsuo Higuchi ${ }^{3}$ \\ 1 Graduate School of Information Sciences, Tohoku University, \\ Sendai 980-8579 Japan \\ ito@aoki.ecei.tohoku.ac.jp \\ 2 Yamatake Corporation, Isehara 259-1195, Japan \\ 3 Faculty of Engineering, Tohoku Institute of Technology, \\ Sendai 982-8577, Japan
}

\begin{abstract}
This paper proposes an efficient fingerprint recognition algorithm combining phase-based image matching and feature-based matching. The use of Fourier phase information of fingerprint images makes possible to achieve robust recognition for weakly impressed, low-quality fingerprint images. Experimental evaluations using two different types of fingerprint image databases demonstrate efficient recognition performance of the proposed algorithm compared with a typical minutiae-based algorithm and the conventional phase-based algorithm.
\end{abstract}

\section{Introduction}

Biometric authentication has been receiving extensive attention over the past decade with increasing demands in automated personal identification. Biometrics is to identify individuals using physiological or behavioral characteristics, such as fingerprint, face, iris, retina, palm-print, etc. Among all the biometric techniques, fingerprint recognition [1,2] is the most popular method and is successfully used in many applications.

Major approaches for fingerprint recognition today can be broadly classified into feature-based approach and correlation-based approach. Typical fingerprint recognition methods employ feature-based matching, where minutiae (i.e., ridge ending and ridge bifurcation) are extracted from the registered fingerprint image and the input fingerprint image, and the number of corresponding minutiae pairs between the two images is used to recognize a valid fingerprint image [1. Featurebased matching is highly robust against nonlinear fingerprint distortion, but shows only limited capability for recognizing poor-quality fingerprint images with low $\mathrm{S} / \mathrm{N}$ ratio due to unexpected fingertip conditions (e.g., dry fingertips, rough fingertips, allergic-skin fingertips) as well as weak impression of fingerprints.

On the other hand, as one of the efficient correlation-based approaches [3], we have proposed a fingerprint recognition algorithm using phase-based image matching [4] - an image matching technique using the phase components 
in 2D Discrete Fourier Transforms (2D DFTs) of given images - , and developed commercial fingerprint verification units for access control applications [5]. Historically, the phase-based image matching has been successfully applied to high-accuracy image registration tasks for computer vision applications [6, 7, 8. The use of Fourier phase information of fingerprint images makes possible highly reliable fingerprint matching for low-quality fingerprints whose minutiae are difficult to be extracted as mentioned above. However, the performance of the phase-based fingerprint matching is degraded by nonlinear distortions in fingerprint images.

In order to improve matching performance for both fingerprint images with poor image quality and with nonlinear shape distortions, we propose a novel fingerprint recognition algorithm combining phase-based image matching and feature-based matching. In this algorithm, two approaches are expected to play a complementary role and may result in significant improvements of recognition performance. Experimental evaluations using two different types of fingerprint image databases demonstrate efficient recognition performance of the proposed algorithm compared with a typical minutiae-based algorithm and the conventional phase-based algorithm.

\section{Phase-Based Fingerprint Matching}

In this section, we introduce the principle of phase-based image matching using the Phase-Only Correlation (POC) function (which is sometimes called the "phase-correlation function") [6, 7, 8, We also describe the POC-based fingerprint matching algorithm.

\subsection{Fundamentals of Phase-Based Image Matching}

Consider two $N_{1} \times N_{2}$ images, $f\left(n_{1}, n_{2}\right)$ and $g\left(n_{1}, n_{2}\right)$, where we assume that the index ranges are $n_{1}=-M_{1} \cdots M_{1}\left(M_{1}>0\right)$ and $n_{2}=-M_{2} \cdots M_{2}\left(M_{2}>0\right)$ for mathematical simplicity, and $N_{1}=2 M_{1}+1$ and $N_{2}=2 M_{2}+1$. Let $F\left(k_{1}, k_{2}\right)$ and $G\left(k_{1}, k_{2}\right)$ denote the 2D DFTs of the two images. $F\left(k_{1}, k_{2}\right)$ is given by

$$
F\left(k_{1}, k_{2}\right)=\sum_{n_{1}, n_{2}} f\left(n_{1}, n_{2}\right) W_{N_{1}}^{k_{1} n_{1}} W_{N_{2}}^{k_{2} n_{2}}=A_{F}\left(k_{1}, k_{2}\right) e^{j \theta_{F}\left(k_{1}, k_{2}\right)},
$$

where $k_{1}=-M_{1} \cdots M_{1}, k_{2}=-M_{2} \cdots M_{2}, W_{N_{1}}=e^{-j \frac{2 \pi}{N_{1}}}, W_{N_{2}}=e^{-j \frac{2 \pi}{N_{2}}}$, and $\sum_{n_{1}, n_{2}}$ denotes $\sum_{n_{1}=-M_{1}}^{M_{1}} \sum_{n_{2}=-M_{2}}^{M_{2}} \cdot A_{F}\left(k_{1}, k_{2}\right)$ is amplitude and $\theta_{F}\left(k_{1}, k_{2}\right)$ is phase. $G\left(k_{1}, k_{2}\right)$ is defined in the same way. The cross-phase spectrum $R_{F G}\left(k_{1}, k_{2}\right)$ is given by

$$
R_{F G}\left(k_{1}, k_{2}\right)=\frac{F\left(k_{1}, k_{2}\right) \overline{G\left(k_{1}, k_{2}\right)}}{\left|F\left(k_{1}, k_{2}\right) \overline{G\left(k_{1}, k_{2}\right)}\right|}=e^{j \theta\left(k_{1}, k_{2}\right)},
$$

where $\overline{G\left(k_{1}, k_{2}\right)}$ is the complex conjugate of $G\left(k_{1}, k_{2}\right)$ and $\theta\left(k_{1}, k_{2}\right)$ denotes the phase difference $\theta_{F}\left(k_{1}, k_{2}\right)-\theta_{G}\left(k_{1}, k_{2}\right)$. The POC function $r_{f g}\left(n_{1}, n_{2}\right)$ is the $2 \mathrm{D}$ Inverse DFT (2D IDFT) of $R_{F G}\left(k_{1}, k_{2}\right)$ and is given by 


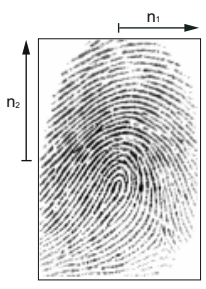

(a)

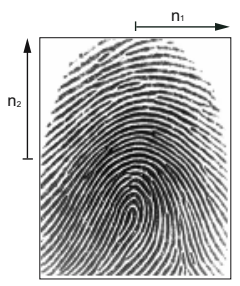

(b)

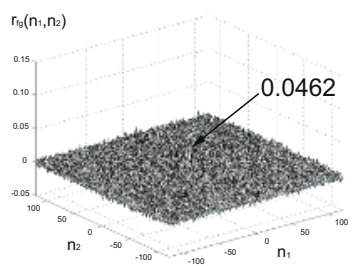

(c)

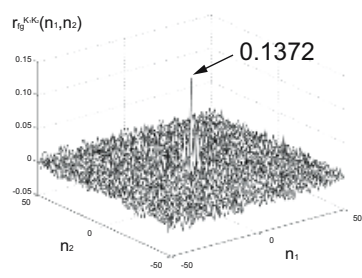

(d)

Fig. 1. Example of genuine matching using the original POC function and the BLPOC function: (a) registered fingerprint image $f\left(n_{1}, n_{2}\right)$, (b) input fingerprint image $g\left(n_{1}, n_{2}\right)$, (c) POC function and (d) BLPOC function with $K_{1} / M_{1}=K_{2} / M_{2}=0.48$

$$
r_{f g}\left(n_{1}, n_{2}\right)=\frac{1}{N_{1} N_{2}} \sum_{k_{1}, k_{2}} R_{F G}\left(k_{1}, k_{2}\right) W_{N_{1}}^{-k_{1} n_{1}} W_{N_{2}}^{-k_{2} n_{2}},
$$

where $\sum_{k_{1}, k_{2}}$ denotes $\sum_{k_{1}=-M_{1}}^{M_{1}} \sum_{k_{2}=-M_{2}}^{M_{2}}$. When two images are similar, their POC function gives a distinct sharp peak. When two images are not similar, the peak drops significantly. The height of the peak gives a good similarity measure for image matching, and the location of the peak shows the translational displacement between the images.

We modify the definition of POC function to have a BLPOC (Band-Limited Phase-Only Correlation) function dedicated to fingerprint matching tasks. The idea to improve the matching performance is to eliminate meaningless high frequency components in the calculation of cross-phase spectrum $R_{F G}\left(k_{1}, k_{2}\right)$ depending on the inherent frequency components of fingerprint images [4]. Assume that the ranges of the inherent frequency band are given by $k_{1}=-K_{1} \cdots K_{1}$ and $k_{2}=-K_{2} \cdots K_{2}$, where $0 \leq K_{1} \leq M_{1}$ and $0 \leq K_{2} \leq M_{2}$. Thus, the effective size of frequency spectrum is given by $L_{1}=2 K_{1}+1$ and $L_{2}=2 K_{2}+1$. The BLPOC function is given by

$$
r_{f g}^{K_{1} K_{2}}\left(n_{1}, n_{2}\right)=\frac{1}{L_{1} L_{2}} \sum_{k_{1}, k_{2}}^{\prime} R_{F G}\left(k_{1}, k_{2}\right) W_{L_{1}}^{-k_{1} n_{1}} W_{L_{2}}^{-k_{2} n_{2}},
$$

where $n_{1}=-K_{1} \cdots K_{1}, n_{2}=-K_{2} \cdots K_{2}$, and $\sum_{k_{1}, k_{2}}^{\prime}$ denotes $\sum_{k_{1}=-K_{1}}^{K_{1}} \sum_{k_{2}=-K_{2}}^{K_{2}}$. Note that the maximum value of the correlation peak of the BLPOC function is always normalized to 1 and does not depend on $L_{1}$ and $L_{2}$. Figure 1 shows an example of genuine matching using the original POC function $r_{f g}$ and the BLPOC function $r_{f g}^{K_{1} K_{2}}$. The BLPOC function provides the higher correlation peak and better discrimination capability than that of the original POC function.

\subsection{Fingerprint Matching Algorithm Using BLPOC Function}

This section describes a fingerprint matching algorithm using BLPOC function. The algorithm consists of the three steps: (i) rotation and displacement alignment, (ii) common region extraction and (iii) matching score calculation with precise rotation. 


\section{(i) Rotation and displacement alignment}

We need to normalize the rotation and the displacement between the registered fingerprint image $f\left(n_{1}, n_{2}\right)$ and the input fingerprint image $g\left(n_{1}, n_{2}\right)$ in order to perform the high-accuracy fingerprint matching. We first normalize the rotation by using a straightforward approach as follows. We first generate a set of rotated images $f_{\theta}\left(n_{1}, n_{2}\right)$ of the registered fingerprint $f\left(n_{1}, n_{2}\right)$ over the angular range $-50^{\circ} \leq \theta \leq 50^{\circ}$ with an angle spacing $1^{\circ}$. The rotation angle $\Theta$ of the input image relative to the registered image can be determined by evaluating the similarity between the rotated replicas of the registered image $f_{\theta}\left(n_{1}, n_{2}\right)$ $\left(-50^{\circ} \leq \theta \leq 50^{\circ}\right)$ and the input image $g\left(n_{1}, n_{2}\right)$ using the BLPOC function. Next, we align the translational displacement between the rotation-normalized image $f_{\Theta}\left(n_{1}, n_{2}\right)$ and the input image $g\left(n_{1}, n_{2}\right)$. The displacement can be obtained from the peak location of the BLPOC function between $f_{\Theta}\left(n_{1}, n_{2}\right)$ and $g\left(n_{1}, n_{2}\right)$. Thus, we have normalized versions of the registered image and the input image, which are denoted by $f^{\prime}\left(n_{1}, n_{2}\right)$ and $g^{\prime}\left(n_{1}, n_{2}\right)$. In practical situation, we store in advance a set of rotated versions of the registered image into a memory in order to reduce the processing time.

\section{(ii) Common region extraction}

Next step is to extract the overlapped region (intersection) of the two images $f^{\prime}\left(n_{1}, n_{2}\right)$ and $g^{\prime}\left(n_{1}, n_{2}\right)$. This process improves the accuracy of fingerprint matching, since the non-overlapped areas of the two images become uncorrelated noise components in the BLPOC function. In order to detect the effective fingerprint areas in the registered image $f^{\prime}\left(n_{1}, n_{2}\right)$ and the input image $g^{\prime}\left(n_{1}, n_{2}\right)$, we examine the $n_{1}$-axis projection and the $n_{2}$-axis projection of pixel values. Only the common effective image areas, $f^{\prime \prime}\left(n_{1}, n_{2}\right)$ and $g^{\prime \prime}\left(n_{1}, n_{2}\right)$, with the same size are extracted for the use in succeeding image matching step.

\section{(iii) Matching score calculation with precise rotation}

The phase-based image matching is highly sensitive to image rotation. Hence, we calculate the matching score with precise correction of image rotation. We generate a set of rotated replicas $f_{\theta}^{\prime \prime}\left(n_{1}, n_{2}\right)$ of $f^{\prime \prime}\left(n_{1}, n_{2}\right)$ over the angular range $-2^{\circ} \leq \theta \leq 2^{\circ}$ with an angle spacing $0.5^{\circ}$, and calculate BLPOC function $r_{f_{\theta}^{\prime \prime} g^{\prime \prime}}^{K_{1} K_{2}}\left(n_{1}, n_{2}\right)$. If the rotation and displacement between two fingerprint images are normalized, the correlation peak can be observed at the center of the BLPOC function. The BLPOC function may give multiple correlation peaks due to elastic fingerprint deformation. Thus, we define the matching score between the two images as the sum of the highest $P$ peaks of the BLPOC function $r_{f_{\theta}^{\prime \prime} g^{\prime \prime}}^{K_{1} K_{2}}\left(n_{1}, n_{2}\right)$, where search area is $B \times B$-pixel block centered at $(0,0)$. In this paper, we employ the parameters $B=11$ and $P=2$. The final score $S_{P}\left(0 \leq S_{P} \leq 1\right)$ of phase-based matching is defined as the maximum value of the scores computed from BLPOC function $r_{f_{\theta}^{\prime \prime} g^{\prime \prime}}^{K_{1} K_{2}}\left(n_{1}, n_{2}\right)$ over the angular range $-2^{\circ} \leq \theta \leq 2^{\circ}$. 


\section{$3 \quad$ Feature-Based Fingerprint Matching}

The proposed feature-based fingerprint matching algorithm extracts the corresponding minutiae pairs between the registered image $f\left(n_{1}, n_{2}\right)$ and the input image $g\left(n_{1}, n_{2}\right)$, and calculates the matching score by block matching using BLPOC. This algorithm consists of four steps: (i) minutiae extraction, (ii) minutiae pair correspondence, (iii) local block matching using BLPOC function, and (iv) matching score calculation.

\section{(i) Minutiae extraction}

We employ the typical minutiae extraction technique [1, which consists of the following four steps: (a) ridge orientation/frequency estimation, (b) fingerprint enhancement and binarization, (c) ridge thinning, and (d) minutiae extraction with spurious minutiae removal. Each extracted minutia is characterized by a feature vector $\boldsymbol{m}_{i}$, whose elements are its $\left(n_{1}, n_{2}\right)$ coordinates, the orientation of the ridge on which it is detected, and its type (i.e., ridge ending or ridge bifurcation). Let $\boldsymbol{M}^{f}$ and $\boldsymbol{M}^{g}$ be sets of minutiae feature vectors extracted from $f\left(n_{1}, n_{2}\right)$ and $g\left(n_{1}, n_{2}\right)$, respectively.

\section{(ii) Minutiae pair correspondence}

A minutia matching technique based on both the local and global structures of minutiae is employed to find corresponding minutiae pairs between $f\left(n_{1}, n_{2}\right)$ and $g\left(n_{1}, n_{2}\right)$ [9]. For every minutia $\boldsymbol{m}_{i}$, we calculate a local structure feature vector $\boldsymbol{l}_{i}$, which described by the distances, ridge-counts, directions and radial angles of the minutia relative to each of two nearest-neighbor minutiae and the types of these minutiae. Let $\boldsymbol{L}^{f}$ and $\boldsymbol{L}^{g}$ be sets of local structure feature vectors calculated from $\boldsymbol{M}^{f}$ and $\boldsymbol{M}^{g}$, respectively. We perform minutiae matching between $\boldsymbol{M}^{f}$ and $\boldsymbol{M}^{g}$ by using their local structure information $\boldsymbol{L}^{f}$ and $\boldsymbol{L}^{g}$, and find the best matching minutiae pair $\left(\boldsymbol{m}_{i_{0}}^{f}, \boldsymbol{m}_{j_{0}}^{g}\right)$, which is called reference minutiae pair. All other minutiae are aligned based on this reference minutiae pair by converting their coordinates to the polar coordinate system with respect to the reference minutia. Thus, we have the aligned minutiae information $\boldsymbol{M}^{\prime f}$ and $\boldsymbol{M}^{\prime}$. For every aligned minutia $\boldsymbol{m}_{i}^{\prime f} \in \boldsymbol{M}^{\prime f}$ ( or $\boldsymbol{m}^{\prime g} \in \boldsymbol{M}^{\prime g}$ ), we calculate a global feature vector $\boldsymbol{g}_{i}^{f}$ (or $\boldsymbol{g}_{j}^{g}$ ), which is described by the distance, direction and radial angle of the minutia relative to the reference minutia $\boldsymbol{m}_{i_{0}}^{f}$ (or $\boldsymbol{m}_{j_{0}}^{g}$ ). Based on the distance $\left|\boldsymbol{g}_{i}^{f}-\boldsymbol{g}_{j}^{g}\right|$, we can now determine the correspondence between the minutiae pair $\boldsymbol{m}_{i}^{\prime f}$ and $\boldsymbol{m}_{j}^{\prime g}$. As a result, we obtain a set of the corresponding minutiae pairs between $\boldsymbol{M}^{\prime f}$ and $\boldsymbol{M}^{g}$ as well as the matching score $S_{\text {minutiae }}\left(0 \leq S_{\text {minutiae }} \leq 1\right)$ defined as

$$
S_{\text {minutiae }}=\frac{(\# \text { of corresponding minutiae pairs })^{2}}{\left|\boldsymbol{M}^{\prime f}\right| \times\left|\boldsymbol{M}^{\prime g}\right|} .
$$

\section{(iii) Local block matching using BLPOC function}

When the number of corresponding minutiae pairs is greater than 2 , we extract local binary images, from $f\left(n_{1}, n_{2}\right)$ and $g\left(n_{1}, n_{2}\right)$, centered at the corresponding 


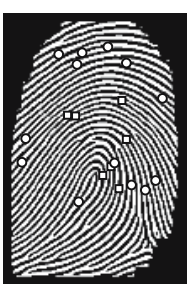

(a)

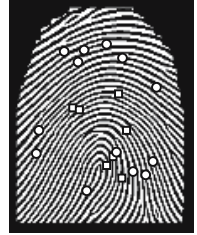

(b)

\section{จा nोII}

(c)

Fig. 2. Example of local block matching using BLPOC function for a genuine pair $\left(S_{\text {minutiae }}=0.41\right.$ and $\left.S_{\text {block }}=0.57\right)$ : (a) binarized registered image, (b) binarized input image, (c) a pair of block around corresponding minutiae (the score of local block matching is 0.59). The symbols $\circ$ and $\square$ denote the corresponding minutiae.

minutiae. The size of local binary image is $l \times l$ pixels, where we use $l=31$ in our experiments. For every pair of local binary images, we align image rotation using the information of minutiae orientation, and calculate the BLPOC function between the local image blocks to evaluate the local matching score as its correlation peak value. The score of block matching $S_{\text {block }}\left(0 \leq S_{\text {block }} \leq 1\right)$ is calculated by taking an average of the highest three local matching scores. On the other hand, when the number of corresponding minutiae pairs is less than 3 , we set $S_{\text {block }}=0$. Figure 2 shows an example of local block matching using BLPOC function for a genuine pair.

\section{(iv) Matching score calculation}

The combined score $S_{F}\left(0 \leq S_{F} \leq 1\right)$ of feature-based matching is calculated from $S_{\text {minutiae }}$ and $S_{\text {block }}$ as follows:

$$
S_{F}= \begin{cases}1 & \text { if } S_{\text {minutiae }} \times S_{\text {block }}>T_{F} \\ S_{\text {minutiae }} \times S_{\text {block }} & \text { otherwise, }\end{cases}
$$

where $T_{F}$ is a threshold.

\section{Overall Recognition Algorithm}

In this section, we describe a fingerprint recognition algorithm combining phasebased image matching and feature-based matching. Figure 3 shows the flow diagram of the proposed fingerprint recognition algorithm.

\section{(I) Classification}

In order to reduce the computation time and to improve the recognition performance, we introduce the rule-based fingerprint classification method [1 before matching operation. In our algorithm, we classify the fingerprints into 7 categories: "Arch", "Left Loop", "Right Loop", "Left Loop or Right Loop", "Arch or Left Loop", "Arch or Right Loop", and "Others". If the two fingerprints to be verified fall into different categories, we give the overall score $S=0$, otherwise matching operation is performed to evaluate the overall score. 


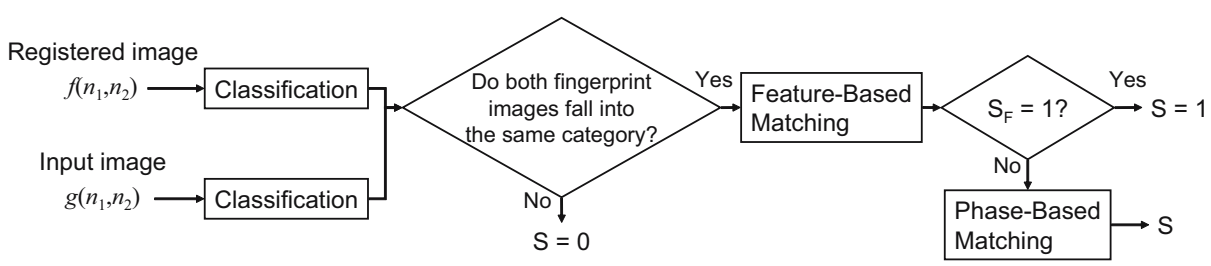

Fig. 3. Flow diagram of the proposed algorithm

\section{(II) Feature-based matching}

This stage evaluates the matching score $S_{F}$ of feature-based matching as described in section 3. If $S_{F}=1$, then we set the overall score as $S=1$ and terminate matching operation, otherwise we proceed to the stage (III).

\section{(III) Phase-based matching}

This stage evaluates the matching score $S_{P}$ of phase-based fingerprint matching as described in section 2. Then, the overall matching score $S$ is computed as a linear combination of $S_{F}$ and $S_{P}$, given by

$$
S=\alpha \times S_{F}+(1-\alpha) \times S_{P},
$$

where $0 \leq \alpha \leq 1$. In our experiments, we employ $\alpha=0.5$.

\section{Experimental Results}

This section describes a set of experiments, using our original database (DB_A) collecting low-quality fingerprint images and the FVC 2002 DB1 set A [10] (DB_B), for evaluating fingerprint matching performance of the proposed algorithm. The following experiments are carried out for the two databases.

A set of fingerprint images in DB_A is captured with a pressure sensitive sensor (BLP-100, BMF Corporation) of size $384 \times 256$ pixels, which contains 330 fingerprint images from 30 different subjects with 11 impressions for each finger. In the captured images, 20 of subjects have good-quality fingerprints and the remaining 10 subjects have low-quality fingerprints due to dry fingertips (6 subjects), rough fingertips (2 subjects) and allergic-skin fingertips (2 subjects). Thus, the test set considered here is specially designed to evaluate the performance of fingerprint matching under difficult condition. We first evaluate genuine matching scores for all possible combinations of genuine attempts; the number of attempts is ${ }_{11} C_{2} \times 30=1650$. Next, we evaluate impostor matching scores for impostor attempts: the number of attempts is ${ }_{30} C_{2}=435$, where we select a single image (the first image) for each fingerprint and make all the possible combinations of impostor attempts.

A set of fingerprint images in DB_B is captured with an optical sensor (Touch View II, Identx Incorporated) of size $388 \times 374$ pixels, which contains 800 fingerprint images from 100 different subjects with 8 impressions for each finger. We first evaluate genuine matching scores for all possible combinations of genuine attempts; the number of attempts is ${ }_{8} C_{2} \times 100=2800$. Next, we evaluate impostor 


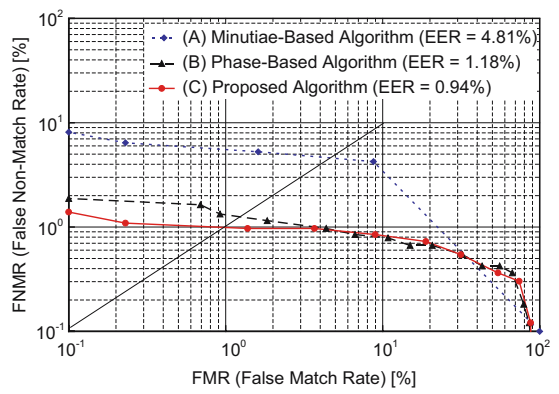

(a)

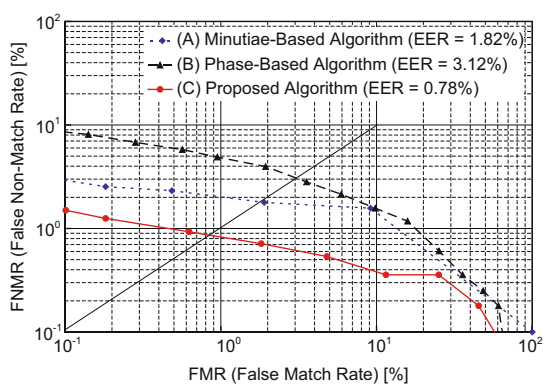

(b)

Fig. 4. ROC curves and EERs: (a) DB_A and (b) DB_B

matching scores for impostor attempts: the number of attempts is ${ }_{100} C_{2}=4950$, where we select a single image (the first image) for each fingerprint and make all the possible combinations of impostor attempts.

We compare three different matching algorithms: (A) a typical minutiae-based algorithm (which is commercially available), (B) a phase-based algorithm described in section 2, and (C) the proposed algorithm. In our experiments, the parameters of BLPOC function are $K_{1} / M_{1}=K_{2} / M_{2}=0.40$ for DB_A and $K_{1} / M_{1}=K_{2} / M_{2}=0.48$ for DB_B. The threshold value for feature-based matching is $T_{F}=0.046$ for DB_A and $T_{F}=0.068$ for DB_B.

The performance of the biometrics-based identification system is evaluated by the Receiver Operating Characteristic (ROC) curve, which illustrates the False Match Rate (FMR) against the False Non-Match Rate (FNMR) at different thresholds on the matching score. Figures 4 (a) and (b) show the ROC curve for the three algorithms (A)-(C) for DB_A and DB_B, respectively. In both cases, the proposed algorithm $(\mathrm{C})$ exhibits significantly higher performance, since its ROC curve is located at lower FNMR/FMR region than those of the minutiaebased algorithm (A) and the phase-based algorithm (B).

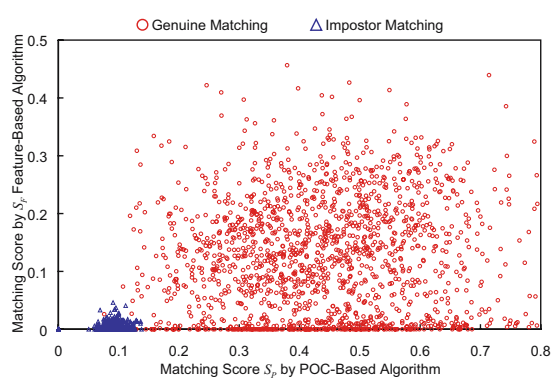

(a)

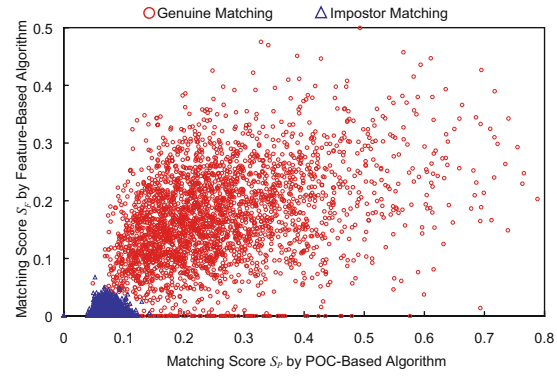

(b)

Fig. 5. Overall joint distribution of matching scores for phase-based matching $S_{P}$ and feature-based matching $S_{F}$ : (a) DB_A and (b) DB_B 
The Equal Error Rate (EER) is used to summarize performance of a verification system. The EER is defined as the error rate where the FNMR and the FMR are equal. As for DB_A, EER of the proposed algorithm (C) is 0.94\%, while EERs of the phase-based algorithm (B) and the minutiae-based algorithm (A) are $1.18 \%$ and $4.81 \%$, respectively. As for DB_B, EER of the proposed algorithm (C) is $0.78 \%$, while EERs of phase-based algorithm (B) and the minutiae-based algorithm (A) are $3.12 \%$ and $1.82 \%$, respectively. As is observed in the above experiments, the combination of phase-based matching and feature-based matching is highly effective for verifying low-quality difficult fingerprints.

Figure 5 (a) and (b) show the joint distribution of matching scores for phasebased matching $S_{P}$ and feature-based matching $S_{F}$. Although we can observe weak correlation between $S_{P}$ and $S_{F}$, both figures (a) and (b) show wide distributions of matching scores. This implies independent matching criteria used in phase-based and feature-based approaches can play a complementary role for improving overall recognition performance.

\section{Conclusion}

This paper has proposed a novel fingerprint recognition algorithm, which is based on the combination of two different matching criteria: (i) phase-based matching and (ii) feature-based matching. Experimental results clearly show good recognition performance compared with a typical minutiae-based fingerprint matching algorithm. In our previous work, we have already developed commercial fingerprint verification units for access control applications [5], which employs specially designed ASIC [11] for real-time phase-based image matching. The algorithm in this paper could be easily mapped onto our prototype hardware, since the computational complexity of feature-based matching algorithm is not significant.

\section{References}

1. Maltoni, D., Maio, D., Jain, A.K., Prabhakar, S.: Handbook of Fingerprint Recognition. Springer (2003)

2. Wayman, J., Jain, A., Maltoni, D., Maio, D.: Biometric Systems. Springer (2005)

3. Venkataramani, K., Vijayakumar, B.V.K.: Fingerprint verification using correlation filters. Lecture Notes in Computer Science 2688 (2003) 886-894

4. Ito, K., Nakajima, H., Kobayashi, K., Aoki, T., Higuchi, T.: A fingerprint matching algorithm using phase-only correlation. IEICE Trans. Fundamentals E87-A (2004) 682-691

5. http://www.aoki.ecei.tohoku.ac.jp/poc.html. Products using phase-based image matching

6. Kuglin, C.D., Hines, D.C.: The phase correlation image alignment method. Proc. Int. Conf. on Cybernetics and Society (1975) 163-165

7. Takita, K., Aoki, T., Sasaki, Y., Higuchi, T., Kobayashi, K.: High-accuracy subpixel image registration based on phase-only correlation. IEICE Trans. Fundamentals E86-A (2003) 1925-1934 
8. Takita, K., Muquit, M.A., Aoki, T., Higuchi, T.: A sub-pixel correspondence search technique for computer vision applications. IEICE Trans. Fundamentals E87-A (2004) 1913-1923

9. Jiang, X., Yau, W.Y.: Fingerprint minutiae matching based on the local and global structures. International Conference on Pattern Recognition. 2 (2000) 1038-1041

10. http://bias.csr.unibo.it/fvc2002. Fingerprint verification competition 2002

11. Morikawa, M., Katsumata, A., Kobayashi, K.: An image processor implementing algorithms using characteristics of phase spectrum of two-dimensional Fourier transformation. Proc. IEEE Int. Symp. Industrial Electronics 3 (1999) 1208-1213 